\title{
I. The electrical properties of pure substances.-Part I. The preparation of Pure Nitrogen and attempts to condense it
}

\section{Professor R. Threlfall}

To cite this article: Professor R. Threlfall (1893) I. The electrical properties of pure substances.-Part I. The preparation of Pure Nitrogen and attempts to condense it, Philosophical Magazine Series 5, 35:212, 1-35, DOI: $10.1080 / 14786449308620375$

To link to this article: http://dx.doi.org/10.1080/14786449308620375

曲 Published online: 08 May 2009.

Submit your article to this journal $\pi$

Џll Article views: 2

Q View related articles $₫$ 
LONDON, EDINBURGH, AND DUBLIN

\title{
PIILLSOPHICAL MAGAZINE
}

\author{
AND \\ JOURNAL OF SCIENCE. \\ [EIFTH SERLES.] \\ $J A N U A R Y 1893$.
}

I. The Electrical Properties of Pure Substances.-Part I. The Preparation of Pure Nitrogen and attempts to Condense $i t$. By Professor R. Threlfald*.

[Plate I.]

N 1886 a paper was published in the Proceedings of the 1 Royal Society by Professor J. J. Thomson and myself, "On an Effect produced by the passage of an Electric Discharge through Pure Nitrogen." The effect in question was briefly as follows:- When a nitrogen tube provided with a small mercury or sulphuric-acid gauge is exhausted to a pressure of about 8 millim. of mercury and then sparked with a discharge too small to heat the tube in a sensible manner, a diminution of the elastic force of the enclosed gas is observed. This diminution was found to be independent of the size or material of the electrodes, of the volume of the tube or the extent of its surface, but to depend on the kind of discharge employed, and its duration; on the temperature and pressure of the gas in the tube during the process of sparking and afterwards. Arguing by exclusion, we attributed the diminution of pressure to a condensation of nitrogen molecules, similar to the condensation which oxygen undergoes when converted into ozone.

This property of nitrogen, if established, would be of undoubted interest, and consequently in $1889 \mathrm{I}$ undertook a

* Communicated by the Author.

Phil. Mag. S. 5. Vol. 35. No. 212. Jan. 1893. 
repetition of the experiments with a view to testing our conclusions by an extended examination of the phenomena in question. This investigation has proved a most laborious task; and it was not until December 1891 that I finally satisfied myself that the effect was due to the combination of nitrogen with the mercury of the pressure-gange under the intuence of the electric discharge, and in the presence, as I think, of a minute trace of some other substance whose nature I have not been able to determine.

The experiments to be described leave little doubt that pure nitrogen at ordinary temperatures does not condense in the same way that oxygen condenses, whether the discharge be by means of external electrodes or by means of wires fused into the tube. Since these experiments form the startingpoint of other researches, I will begin by an account of a method by which pure nitrogen may be most advantageously prepared. Singularly enough this apparently simple chemical problem has not, so far as I know, been solved hitherto. Stas, in his researches, makes the remark that nitrogen is easily obtained pure (Bulletin de l'Académie Royale des Sciences de Belgique, 1860, sér. 2, t. x. p. 254); and this remark may very possibly have produced misunderstanding--everything depends on what is meant by the word pure. 'The fundamental principles in all processes of purification are :-1st, that reagents used to remove any impurity must really keep the impurity when they have got it, and not liberate it or its equivalent through any instability of the comjoounds formed; and 2 nd, that the reagents themselves must not give rise to impurities. As an example of a common violation of the first principle, I will mention the absorption of sulphuretted hydrogen, sulphur dioxide, or the oxides of nitrogen, by potash or soda ; and of the second, the use of imperfectly prepared phosphorus pentoxide. Trouble arising from layers of air or other gases condensed on the surfaces of glass vessels can only be relieved by arranging the method of production of the gas required in such a way that the glass surfaces may remain for weeks or month in contact with the gas nnder experiment, otherwise completely purified. If mercury be in contact with glass in any part of the apparatus, then, as I believe, it is impossible by ordinary means to be sure that the glass is ever completely denuded of its primitive layers of gas. I append a list of some of the various ways in which nitrogen has been prepared for careful work, and shall, I think, be able to show that all these processes are open to objection, at all events where a continuous supply of gas is necessary. The list is short, because nearly all experimenters simply state that 
they used pure nitrogen without giving details of its method of preparation.

Name. Reference.

J. S. Stas... Bull de l'Acad. de Belgique, vol: $\mathrm{x}$. p. 254 .

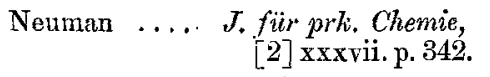

Olzewski .... Wien. Anzeiger,

March 1884.

Wroblewski.. C. R. xcviii. p. 982

Lupton ...... C. S. J. 1876;

Chem. News,

xxxiii. p. 90.

H. Deslandres. C.R. ci. p. 1256.

W. Gibbs.... Ber, x. p. 1387.

N. W. Fisher. Pogg. Ann. xvii. p. 137.

Regnault .... C. R. t. xx. p. 975 .

Wiillner .... Pogg. Ann. cxlvii. p. 325 .

Huggins ... P Phil. Trans. 1860.

Warburg .... Ann. der Chem. und der Phys. 1887, p. 548 .

\section{Method.}

By passing purified air over hot copper turnings and gauze, which are previously reduced by hydrogen. Copper oxide at end of tube (?); ordinary absorbent reagents to help purify the gas after leaving the hot copper.

From chloride-of-lime cubes (made by Winkler's method) and aqueous ammonia (see C. S. J. 1887 , p. 442).

Hot copper and air.

Hot copper and air.

Air and ammonia over hot copper; claims to get pure $\mathrm{N}$ with a short tube.

Hot copper and air; no precaution mentioned.

Sodium nitrite, ammonium sulphate, and potassium dichromate in excess. If there is a trace of cbloride present, impurities are introduced.

Uses cuprous chloride as an absorber of oxygen from air.

Hot copper and air.

Burning phosphorus in air over mercury and removing traces of oxygen by incandescent iron wire.

Purified air over hot reduced copper.

Hittorf's method. Dust-free air over hot white phosphorus, then over potassium permanganate, solid potash, and phosphorus pentoxide. Special drying in some cases followed by exposure (Wied. Ann. xl. p. I) to nascent sodium obtained by the electrolysis of glass.

It is clear that the most popular way of making nitrogen is to absorb the oxygen from air by means of red-hot copper. There are a great many objections to this method, however, even when precautions are taken against the possibility of the formation of oxides of nitrogen. In the first place, a satisfactory method of making connexion to the porcelain tube is unknown to me; indiarubber stoppers are undesirable in any B 2 
case, and particularly in places where they can become hot. One never feels certain, moreover, that a red-hot tube, even of glazed porcelain, is absolutely impermeable to furnacegases. If one uses a bath of magnesia this difficulty may perhaps be overeome, but there remains the difficulty of making proper connexions.

If hard glass be employed instead of porcelain, then there is insecurity in the joints between it and the glass nsed for the remainder of the apparatns. Metal tubes present similar difficulties with regard to the junctions, and are besides more or less porous at high temperatures. Another grave disadvantage is that, whether porcelain or glass tubes be used, there is always a chance of a crack occurring, and perhaps escaping notice.

The reduction of the copper also presents great difficulty: it is more difficult to get a strong stream of really pure hydrogen or carbon monoxide than to get the nitrogen; and any sulphur absorbed by the copper is a permanent disadvantage; for, as metallurgists know, copper containing sulphur may be oxidized and reduced many times and yet at each oxidation some sulphur will burn out. The reason is, of course, that sulphide of copper heated in air forms some sulphate of copper as well as sulphur dioxide. On the next reduction sulphide of copper is re-formed, and then, on passing air over the mixture, the process of incomplete oxidation is repeated. Judging by a disenssion which took place in Section A of the British Association not long ago, these simple facts are not as widely known as might have been expected. I know of no reagent which will absorb sulphur dinxide so as to form an absolutely stable compound, and which is itself easy to prepare in a state of sufficient purity to be above suspicion of giving off foreign matters to the nitrogen. Of course it may be argued that a large number of vessels containing, say, a solution of caustic potash might be used, the second retaining the sulphur dioxide given off from the first, and so on; but this device is obviously unsuited for a continuous process, where the reagents must be untouched for weeks or months.

Finally, I gave the process a careful trial, and found it unsatisfactory from the causes mentioned; and, as a matter of fact, the trouble of preparing hydrogen or carbon monoxide in a sufficient state of purity to reduce the copper without contaminating it with sulphur or chlorine is at least as great as the trouble of preparing nitrogen itself.

With regard to the method of passing air over melted white phosphorus, the manipulation of phosphorus is always 
troublesome, and I have not been able to find any information as to whether phosphorus absorbs gases which might be given off during the process of the removal of oxygen from air. Warburg used a solution of potassium permanganate to absorb the vapour of phosphorus; but I have not been able to obtain information as to whether such a solution is to be relied on, in the first place to remove all the phosphorus, and in the second to give off nothing else during the process. I understand, from the paper in Wied. Ann. vol. xl. 1890, p. 1, that Warburg, in his later experiments, did not succe?d to his satisfaction in removing the last trace of oxygen by this method, but had to electrolyse the glass of his vaenum-tubes so as to liberate the sodium in presence of the nitrogen, in order to get rid of the last traces of oxygen and hydrogen. This method appears satisfactory if one has at one's disposal a sufficient electromotive force, suitable glass, and an infinite time : the hydrogen appears to be absorbed as by palladium.

Another method over which I spent six months of fruitless labour is based on the decomposition of ammonium bichromate by heat. This method is convenient, because if the bichromate could be got to deliver nitrogen of suitable quality, small quantities of the salt could be decomposed in bulbs fused on to the vacuum-tubes along with suitable purifying reagents. Ordinary ammonium bichromate (i.e. that sold as pure) gives off a good deal of ammonia and oxides of nitrogen as well as nitrogen itself and water-vapour. This means the use of a good many purifying reagents, so that the method loses its advantages of simplicity; and in addition the oxides of nitrogen cannot be fixed satisfactorily by any suitable reagent known to me. I thought, however, that possibly very pure ammonium bichromate might decompose in a simpler manner, and consequently went to great trouble to procuro a pure sample. For this purpose I purified a large quantity of ammonium chloride and sulphate by the method described by Stas (Bull. de l'Académie Royale de Belgique, 2nd sér. t. x. p. 283), repeating the boiling with nitric acid more often than Stas found requisite. The purified samples were used to generate gaseous ammonia (under the influence of specially prepared marble-lime), which after copions washing was absorbed by chromic acid. Some samples of this ammonia were passed over red-hot platinized asbestos with sufficient pure oxygen* to destroy three quarters of the ammonia, $4 \mathrm{NH}_{3}+3 \mathrm{O}_{2}=6 \mathrm{H}_{2} \mathrm{O}+2 \mathrm{~N}_{2}$, with a view to destroying any traces of organic ammonium compounds; but the

* The method of procuring pure oxygen will be described in another connexion. 
resulting bichromate of ammonium coming from the small quantity of ammonia which was not destroyed by the oxygen did not differ in behaviour from the other samples. It may be noted here that Stas found (loc. cit.) that purified ammonia has a much less unpleasant smell than ordinary ammonia : careful trial, however, failed to enable me to detect any difference between the smell of my purest ammonia and that obtained by boiling so-called pure solution of ammonia obtained from the dealers. I conclude that the "pure ammonia" of commerce is much purer now-a-days than it was thirty years ago.

The chromic acid was made from repeatedly crystallized bichromate of potash, bought as pure, and the sulphuric acid which is sold for analytical purposes. The resulting chromic acid was washed free from sulphuric acid by strong nitric acid, distilled for the purpose. I found incidentally that the addition of a little chromic acid to the mixture of nitric and sulphuric acid in the retort appeared to have a good effect in diminishing the quantity of nitrous acid which generally accompanies "fuming" nitric acid. The washed chromic acid was dried on tiles in the usual way, and heated with great care and continual stirring until it partly melted; but even so it was slightly decomposed. Of course the greater portion of the nitric acid had been previously got rid of by heating the chromic acid on a water-bath in a partly exhausted retort with ground-on condenser, the latter containing some sticks of potash. The chromic acid being partially decomposed gave a solution, which had to be filtered through glass-wool before it was used to absorb the ammonia. This solution was tested by distilling, the distillate being tested for nitrites both before and after reduction with sodium amalgam. The result of these tests was to show that either the solution contained no nitro-compounds, or, if so, not more than are contained in the same quantity of platinum distilled water. The bichromate of ammonia made from this was recrystallized three times from platinum distilled water. Some small samples were orystallized up to six times, but did not differ from the results of the third crystallization. All these samples, when heated either in air or in vacuo, gave off nitrous compounds, and did not behave in any way differently to the sample which had been bought as pure. I therefore conclude that the normal decomposition of ammonium bichromate is complex, and that it is not a good substance to use as a source of nitrogen.

I will now describe a method of procuring nitrogen which has not been mentioned hitherto, which lends itself remarkably well to a continnous process, and which is almost, but not 
quite, free from objection. I refer to the copper-ammonia and chromous-chloride method of Berthelot and Recoura ('Watts' Dictionary,' new edition, and Ann. de Phys. et de Chim. s. 6, vol. x. p. 5). When air is exposed to a large surface of copper wet with strong ammonia, the oxygen is for the most part absorbed. In my experience with the arrangements about to be described, there is only about three per cent. of oxygen left after the reagents have acted for half an hour, and only one per cent, after three hours. Since in prictice with $\mathrm{my}$ apparatus the air often stands over the copper for a day or even a week, the absorption is probably nearly complete. The last traces of oxygen are absorhed by a strong solution of chromous chloride, which perhaps exercises a stronger absorptive power on oxygen than any other liquid, and is for this purpose quite easily prepared. As the method is not so well known as it deserves to be, I give the following description from Recoura's paper :-

A large flask is taken, and in it are placed 250 to 300 grammes of granulated redistilled zine with 50 grammes powdered crystals of potassium bichromate. The bichromate must be finely powdered; it should be pounded till it looks bright yellow. Three hundred cubic centimetres of pure hydrochloric acid are mixed in a beaker with two hundred cubic centim. of water, and I have found it advantageous to heat the mixture almost to boiling. The contents of the beaker are then poured as quickly as possible into the flask, and a violent evolution of hydrogen and steam at once commences. If the flask is too small, some of the liquid will of course be projected from it. Under the powerfully reducing influence of the zinc and hydrochloric acid, the chromium is rapidly reduced to the state of green oxide, which then redissolves to a beautiful blue solution. The whole reaction only takes about ten minutes or a quarter of an hour. If the bichromate is not sufficiently finely powdered, a further quantity of hydrochloric acid may have to be added to complete the reaction, or the mixture may be left (excluding air) for a few hours. The blue solution contains chromous chlom ride, chlorides of zinc and potassium, and possibly various impurities. The slightest trace of oxygen destroys the perfect colour of the chromous chloride by converting it into the green or grey substances investigated by Recoura. On standing over zine for some time, however, if not too much oxidized and slightly acid, the chromium compounds become again reduced and the fine blue colour reappears. The colour is as clear and bright and almost exactly of the same shade as the colour of an ammoniacal solution of cupric hydrate. Recoura states that a stream of gas containing free oxygen 
can be completely deprived of the latter by allowing it to bubble once through a flask containing the blue liquid. To test this I placed two flasks in series, both containing a few hundred centimetres of the solution, and fitted with tubes allowing air to be sucked through both, one after the other. Though I allowed the air to pass much more briskly than if I had been actually using the process (say three times faster than one ordinarily allows the oxygen to pass in an organic analysis), I did not observe any change in the second flask till the first had long been quite opaque and had become more than warm, and then suddenly the contents of the second flask became attacked. In manipulating the chromouschloride solution, it is useful to have a flask of the blue liquid permanently at hand to purify the carbonic acid or nitrogen which must be used in the subsequent operations. In many cases it does not matter whether the gas has a trace of hydrogen in it or not; but for my purpose I was not willing to run any risk of having hydrogen about, and consequently I never allowed the purifying flask to contain zinc.

Having satisfied myself as to the reliability of the chromons chloride as an absorber of oxygen, I undertook a number of experiments with the object of discovering, 1st, the most suitable form in which to use it; 2nd, whether in the process of absorption any other gas was given off, or any vapour which could not be safely absorbed. In these experiments large vessels containing copper and ammonia (arranged in a manner to be presently explained), and capable of furnishing a continuous steam of nitrogen mixed with oxygen in proportions which could be varied at will, were used throughout. The ammonia was removed from the nitrogen by strong sulphuric acid that had been heated to boiling and cooled in a vacuous desiccator. With regard to the best way of using the chromous chloride, I began with the impression that tubes filled with the dry salt would be the most convenient, especially as Recoura shows that small crystals of dry ehromons chloride have an affinity for oxygen which surpasses that of the solution. I therefore prepared large quantities of the blue solution, and obtained the crystals from it by the methoif recommended by Recoura, which is briefly as follows :-The blue liquid is mixed with a saturated solution of soditm acetate, which precipitates chromous acetate in the form of a dark red powder. This powder is then dissolved in dilute bydrochloric acid, and the chromous chloride precipitated from the solution by passing in gaseous hydrochloric scid so as to gradually increase the strength of the hydrochloric-acid solution 
The crystals are repeatedly washed with strong hydrochloric acid and dried by pressing them into tubes with Kieselguhr. All these operations require to be performed in an atmosphere free from oxygen, and consequently are troublesome. The crystals are of a tine sky-blue colour, and have a really extraordinary affinity for oxygen. For my purpose, however, I disliked the use of the sodium acetate, because I never felt quite sure that I had washed all the resulting acetic acid out of the crystals. I have no reason to believe that, even if acetic acid were present during the absorption of oxygen, any organic compound would be libcrated, but in the absence of any information I thought it better not to run the risk. I therefore turned my attention to the original blue solution. This of course contains chlorides of zine and potassium, free hydrochloric acid, hydrogen in solution, and possibly traces of sulphur compounds coming from impurity in the potassium dichromate. It is a question, then, whether during the absorption of oxygen we may not have chlorine or sulphur compounds liberated. Both the acid and zinc were free from arsenic and antimony, so that I had no uneasiness about these substances. I first satisfied myself that chlorine was not given off, by allowing nitrogen with 3 per cent. of oxygen to pass through two flasks of chromous chloride, and then through copper sulphate to retain traces of hydrochloric acid, and finally over paper dipped in a solution of iodide of potassium and starch. Thongh ten litres of gas were passed through, I could detect no change in the starchpaper. Sulphuric acid, when added to the blue liquid, is partly reduced and hydrogen sulphide is given off ; consequently, since potassium bichromate is generally contaminated with potassium sulphate, which is hard to remore, I had to test whether any sulphur compounds were evolved. For this purpose, and with the arrangement of the last experiment, I passed nitrogen through the liquid and then over filter-paper, saturated with a slightly acid solution of nitrate of silver, but no change of colour could be detected. It remained for me to make sure that in the liquid I intended to use there could be no volatile impurity (the traces of bydrochloric acid itself need not be considered, because hydrochloric acid can be stopped readily by potassium hydrate).

Before discussing this I must explain my method of preparing the blue liquid, which was as follows :-About ten litres of the raw product were prepared in several operations and drawn into one large vessel through a filtering-tube. This tube contained a length of four inches of compressed glasswool, and was closed at its lower end by a perforated platinum 
plate whose edges were fused into the tube. The blue liquid was forced through the plug by a pressure of carbonic acid made from marble, which had been boiled for a good many hours at reduced pressure in water before being placed in the Kipp's apparatus. This carbonic acid always passed through a wash-bottle of blue litmus purified in the way to be described by a preliminary experiment. The filter acted so perfectly that the liquid passing throngh it was brilliantly clear and free from zinc-dust, which I greatly feared. In these preliminary operations I was practically forced to make use of some rubber-stoppers, and this is the worst fault of the method. However, all the stoppers used were well coated with pure paraffin; and though they might have given off some hydrocarbons, still I was unable to detect any trace of them in the nitrogen finally obtained. Some rubber-tubing was also employed to make joints, but the same precautions were taken with it, and in all cases the sulphur was well cleaned off. The filtered liquid was finaily got into a retort with a ground-on condenser, and was concentrated to one third of its original volume in a brisk current of carbonic acid.

Towards the end of the operation the stream of carbonic acid was stopped, and a partial vacuum was created in the retort and condenser by a water-pump, so that the final concentration took place under reduced pressure. These precautions were considered sufficient to remove the hydrogen dissolved in the liquid as well as any traces of volatile sulphur compounds resulting from supposed impurities in the potassium bichromate. The resulting liquid was a clear blue syrup, and was transferr ed at once to the absorption-apparatus. Before going into any details as to other precautiens, I will describe the arrangements adopted for the continuous preparation of the nitrogen by this process. These arrangements will be most easily understood by a reference to the diagram (Pl. I.), though taps and clips are exaggerated for clearness. $g$ is a pipe projecting through the window of the laboratory, and terminating close to the wall at a height, of about three metres from the ground. The University paddock separates the laboratory by nearly a quarter of a mile from the nearest road on this side, but the road on the other side lies within 150 yards. The elevation of the laboratory is 120 feet above sea-level. Consequently the composition of the air is probably more nearly that of typical "country air" than of town air. $\mathrm{H}$ is a U-tube with paraffin (M.P. $51^{\circ}$ C.) covered cork stoppers, and is tightly packed with cotton-wool, from which the dust was blown before the tube war mounted. $\mathrm{F}$ is a two-way tap, 
enabling the large glass vessel to be placed in communication either with the external air through $\mathrm{H}$, or with the system of purifying tubes. The large vessel with tap $\mathrm{E}$ is filled with copper ganze and strip, and communicates by a tube having a rubber-joint and clip $\mathrm{D}$ and tap $\mathrm{C}$ with another large vessel placed $2 \cdot 4$ metres above it (measured from bottom to bottom). The upper vessel can be put in communication with a water-pump by a pipe passing through $B$ and having a tap at $A$. This upper vessel contains strong liquid ammonia. It will be readily understood that by working the taps \&c. the anmonia may be drawn into the upper vessel while air is being admitted to the lower, and may then bo allowed to descend by gravity, and furnish the requisite head to force the gas through the remainder of the apparatus when the two-way tap F is suitably turned. G is an auxiliary tap for allowing samples of the gas to be dravin off and tested for oxygen in an ordinary Hempel's gas-analysis apparatus, not shown in the diagran. The air, from which the greater part, of the oxygen has been absorbed by the copper and ammonia, now passes into a horizontal tube I about half filled with strong sulphuric acid, where the greater portion of the ammonia is absorbed. The management of this tube requires care or the entrance soon gets stopped up by the formation of ammonium sulphate. A f'ier two years' work this tube was replaced by a large glass wash-bottle with ground-in stopper. $J$ is a tube containing strong sulphuric acid through which the gas bubbles; $h$ is merely a drawn-out end for facilitating the blowing-out of the joints of the glass tubes. At $\mathrm{K}$ are two "double" wash-bottles containing the chromous chloride syrup; and L represents a U-tube with ground-in tap-stoppers filled with glass beads, and likewise containing chromous chloride. The stoppers of $\mathrm{L}$ and $\mathrm{K}$ are weighted with lead weights to enable them to stand the pressure, and mercury cups are arranged round the stoppers. $\mathrm{M}$ is a tube containing a solution of caustic potash. $\mathrm{N}$ contains nitrate of silver together with a little silver hydroxide and potassium nitrate, $\mathrm{O}$ contains more potash, and $\mathrm{P}$ contains strong sulphuric acid. $Q$ is half filled with potash in lumps, and half with soda-lime. $\mathbf{R}$ is a tube into which phosphorus pentoxide has been distilled so as to present a large surface. S, a Winchester bottle with ground-in stopper, and the wide tube U both contain phosphorus pentoxide. Originally there was a mercury-gauge at $V$, and though this was dispensed with, the apparatus for eliminating mercury vapour was allowed to remain because one or two drops of mercury had got into the tube near V. $W$ is a specially fine tap protected with 
mercury ; and $X$ is a little phosphorus-pentoside tube required to protect the tay, which is lubricated with phosphorus pentoxide, and which of conrse requires to be kept at the proper degree of concentratiou. $\mathrm{Y}$ is a sulphur tube, and at $Z$ are silver and copper tubes. Beyond $Z$ is seen a tube and gauge of the typo used in experimenting on the nitrogen at low temperatures. $\Sigma$ is a small phosphoric-acid guard-tube, $\Psi$ is a sulphur tulee, and $\Omega$ and $\Phi$ are silver and copper tubes separated from each other by long tabes of capillary bore. This system divides at $b$, so that it may be put in communication with a water-pump viâ $d, a$, and $e$, or a Geissler pump through $f^{\prime}$, where there is another tap not shown. The taps at $\mathrm{F}, \mathrm{W}, d$, and $f$ are fine samples of the diagonal taps made by the successors of Geissler, of Bonn; they have all been reground with "optical" precision, and are all lubricated (except F) with phosphorus pentoxide and protected with mercury. The weak point in the system is at the stoppers of $\mathrm{L}$ and $\mathrm{K}$, which had to be lubricated with tallow. In order to satisty myself of the nature of the tallow I began by purifying it with animal charcoal, and finally by keeping it melted for eight hours in a vacuum. After this the smell was almost gone and the tallow was applied to the stoppers mentioned. The tap F was lubricated with vaseline and beeswax also long melted in vacuo. I feared that I should introduce hydrocarbons at these points, but the convenience of using the tallow caused me to try it, and undertake an examination of the gas in a vacuum-tube in order to find whether any bydrocarbons were present or not. The result was that no trace of the "three" bands could be found, so that the enormous complications which would have been required in the other event were happily unnecessary. The stoppers of the large ammonia vessels were of paraffin-coated rubber. It will be noticed that all the points where tallow or rubber were used are to the right of the main purifying system, which includes strong sulphuric acid, and the grinding of all the stoppers was so accurate that an almost inappreciable film of tallow was all that was necessary. It remains to describe the precautions taken with the various reagents, beginning on the right.

Ammonia. -The examination of the ammonia sold as "pure liquid ammonia" which I made in connexion with the ammonium bichromate experiments showed that nothing was to be gained for the purpose of the main experiment by attempting any further purification.

Copper.-At different times sheet gauze, wire, and turnings were employed. The purity of the copper does not 
seem to be of importance, but it is necessary to get rid of grense. This must be done in such a way as not to waste too much copper. In order to clean the turnings and gauze they were soldered into a copper tube, and a considerable quantity of shale benzine was distilled and allowed to condense among them. This secures that every part shall be wetted. The process was then continued by gasoline. The gasoline was distilled over the copper to avoid residues, and was finally dried out at about $120^{\circ}$ for twelve hours, a good stream of filtered air being drawn over the copper by means of an airpump. The same process was gone through with alcohol, and finally water. The copper was then washed with dilate chromic acid and digested with strong ammonia for half an hour. All this trouble was gone to, to avoid wasting the turnings, which one has to cut oneself in order to get them in nice long curls, so that they will stand in the bottle and not break up at once and go to the bottom. After about a year's work it was found necessary to renew the copper and ammonia - the former having almost disappeared in a yellow red mud (cuprous oxide?) which was not further examinedand the ammonia solution had also partially lost its blue colour. Copper strip about 1 millim. thick was now used to replace the gauze and turnings and wire, and was of course strong enough to stand dipping in nitric and sulphuric acid. It is, on the whole, more conrenient to use copper in strip than any other form.

Sulphuric Acid.-This was bought as pure for analysis, and was heated with pure ammonium sulphate for some time before being used. Its purity was, however, of no great moment, except in the case of the final drying-tube P.

Chromous Chloride.-Already discussed.

Potassium Hydrate.-During the experiments on the decomposition of ammonium bichromate, I incidentally noticed that all the samples of potash and soda in my possession yielded traces of nitrites, either on simple solution or when distilled with strong sulphuric acid (which was itself free from this impurity) and the distillate reduced by sodium amalgam. The samples of potash which were said to have been purified by solution in alcohol were the worst in this respect, while some rough lumps of commercial caustic potash were nearly free from the impurity in question. I found considerable quantities of nitrites also in samples of potash procured by exposing clean potassium to moist air. I did not try with dry air. After a good deal of trouble I was reduced to using a solution of a sample of potash in sticks not specially puritied, but which only gave the reaction very 
slightly, and was boiled under diminished pressure for two or three hours before use. The solution still gave a distillate with sulphuric acid which reacted with starch and iodine, but after a time so long that it was possible that the oxposure to air during the experiment had been sufficient to supply the nitrite discovered. In fact a control experiment made with some ammonia, known to be free from nitrites or nitrates, yielded a distillate which coloured to nearly the same extent on standing. The solution was made up to the strength recommended for use in organic analysis.

Silver Nitrute.-Crystals of silver nitrate bought as pure were dissolved in platinum distilled water to make a ten-percent. solution ; to this, when in the tube, a little of the potash solution was added-so as to form a little silver hydroxide. This hydroxide darkened gradually in the bright ligbt to which it was exposed. It was not considered necessary to investigate the solution for traces of free nitrous compounds, as the gas on leaving it passed through another potash-tube.

Solid Potash and Potash-Lime.-The above-mentioned stick potash was used, and the potash-lime was made by adding a little of the solution to some marble-lime prepared in the laboratory; the drying process was carried out in a clean iron dish.

Phosphorus Pentoxide.-This, being the last reagent traversed by the nitrogen, requires to be exceptionally carefully treated. I began by acting on a hint obtained in 1889 from Prof. Josiah P. Cook, of Harvard, and endeavoured to prepare a pure sample of the pentoxide by burning phosphorus in a very strong draught of air, rather than by attempting to purify the ordinary commercial reagent. For this purpose an elaborate sheet-iron cylindrical chamber, measuring 3 feet by 2 feet, was prepared and fitted with suitable contrivances for carrying out the combustion of several pounds of phosphorus. The air-blast was obtained from a Root's blower worked by a gas-engine, and the filtered air travelled through a tin tube about 4 feet long and 7 inches in diameter, filled with calcium chloride in the state usually described as "rough dried." After many attempts' a large quantity of excellent pentoxide was obtained, which, however, though indefinitely better than the commercial product, still retained traces of phosphorus. The tests I employed for the purpose of discovering whether the pentoxide contained free phosphorus or not were the following:-(1) Dissolving 10 or 15 grammes weight of the pentoxide in clear distilled water and observing $(a)$ whether anything remains undissolved, $(\beta)$ whether, at the moment of throwing the phosphorus pentoxide on the 
water any smell of phosphorus can be detected. $(\beta)$ is a good deal more delicate than $(\alpha)$, for I clearly discerned the smell of phosphorus after the pentoxide had become pure enough to yield no perceptible particles when thrown into water. (2) A small porcelain crucible was filled with the pentoxide to be tested, the lid was replaced, and the crucible rapidly raised to a dull red heat, or till the pentoxide just began to distil off. The room in which the operation was conducted was quite dark except for the fickle light emitted by the Bunsen flame used in heating the crucible. As soon as the proper moment arrived the observer adjusted the crucible tongs with one hand and turned out the Bunsen with the other. The cover of the crucible was then removed, and by turning on a tap ready to hand a jet of oxygen was caused to impinge on the phosphorus pentoxide in the crucible: if any phosphorus is present "sparkles" are seen on the surface of the pentoxide; and if the room is dark and the eyes of the observer sensitive this is a very good test, but not quite so good as that by the smell caused by throwing a large quantity of the pentoxide on water. (3) A few drops of a solution of chloride of gold in ether were mixed with about 500 cub. centim. of water, so as to make a very dilute solution. This solution was divided into two parts and occupied two similar cylinders. To one portion the solution obtained by throwing about 1 gramme of the pentoxide into 100 cub. centim. of water was added, and to the other an equal quantity of pure water. The observation, lasting for several days, consisted in watching the cylinders and noting the reduction of gold that took place, as evidenced by the rosy purple colour which the solution assumed. This test is not so delicate as the others, and is valueless unless a control experiment is made in the manner here described.

As tested by these tests, the phosphorus pentoxide prepared by myself was never quite free from phosphorus, and I was therefore obliged to undertake the distillation of the raw product in a stream of oxygen. This of course involved the making of the oxygen in a sufficient state of purity. Fortunately the presence of nitrogen in oxygen used for this purpose is of no consequence so long as the amount is not large, the impurities to be dreaded are hydrocarbons and chlorine. The oxygen was consequently prepared from potassium chlorate with the usual precautions as for an organic analysis, being passed through several potash-tubes and washbottles on its way to the gas-holder-a large copper one free from grease on the taps. From the gas-holder the oxygen passed through the following system :- 
(1) Solution of potassium hydrate in tube 4 feet long.

*(2) Tube 18 in. long, first half filled with metallic antimony, second half filled with clean silver foil.

(3) U-tube $12 \times 1$ in., solution of silver nitrate.

(4) U-tube $12 \times 1$ in., solid potash.

(5) 2 ditto, snIphtric-acid beads.

(6) 1 bottle, phosphorus pentoxide.

(7) 2 U-tubes, ditto.

All the connexions were of glass throughout, but it was found convenient to use several rubber stoppers coated with hard paraffin. The distillation of the pentoxide was carried on in a porcelain tube 2 centimetres in diameter and nearly a metre long, the connexions being made with glass tubes pushed into the ends of the porcelain tube, and packed with glass-wool and some pentoxide. This packing is very firm and good. The first three quarters of the porcelain tube were nearly filled with pentoxide, the last quarter contained platinized porcelain and asbestos, the latter most carefully purified by boiling in hydrochloric acid and strong heating. The distillation was carried on in some cases so as to cause the pentoxide to condense in the tubes and vessels in which it was to be used, and a considerable quantity was also collected in a bottle.

The final product passed all the tests I have enumerated, and was used throughout the nitrogen apparatus, even for drying the pump, lubricating the taps, \&c. Of course a good many tubes full of pentoxide were distilled, and nearly three months were consumed in obtaining the final product. If I had not had the assistance of Mr. J. A. Pollock in this work, I have an impression that I might be at it still. The commercial pentoxide was used in this work, for the substance we prepared ourselves was probably good enough for most purposes as it was. I have sometimes wondered whether experimenters who have of late used so much pentoxide for critical work on gases have recognized the necessity of testing the product obtained by distilling the pentoxide in oxygen. In my experience, unless the oxygen stream be very strong it is quite possible to fail to remove the last traces of free phosphorus.

Sulphur†.-I happened to have some very pure sulphur which had been prepared for another purpose. It will be

* It was found as the result of several preliminary experiments that metallic antimony is a rather good absorber of chlorine, and keeps what it absorbs.

$\dagger$ An account of the experiments with sulphur will form a chapter of this monograph. 
sufficient here to say that the sulphur was originally precipitated from calcium polysulphides by hydrochloric acid ; it was laboriously ground and washed, and distilled five times, and care was taken not to let it take fire. The degree of purity attained was tested by burning off large quantities in a burnished platinum dish, and continuing the process till the residue had disappeared. This product was rather precious, consequently it was made the most of in the present case by using it to coat glass beads : some in powder was also employed near the entrance of the purifying tubes.

Copper and Silver-Both bought as pure and deprived of grease by dipping in appropriate acid solutions.

General Remarks.-During the two years the apparatus has been in use various accidents, such as the breaking of pumps \&c., have at different times caused some of the reagents to get mixed up, and the apparatus has been taken down and set up again some three or four times; the first bottles of chromous chloride have also required to be refilled. The phosphorus pentoxide has never required to be touched. I wish to draw attention to a remark of Hittorf's as to the importance of using tubing free from air-bubbles. On several occasions I have been much troubled by the minute leaks which such imperfect tubes give rise to at the joints, and $I$ have in my possession at least two tubes leaking from this cause, but so minutely that even when the leak was localized to within six or seren inches it was impossible to say exactly where it was. In work of this kind the cleaning of the tubes is of course of great importance. If the work is to be done rapidly the great secret is to clean the tube with alcohol before the jointing or general glass-blowing operations are carried out on it. The dirty places are almost always near where the blowpipe-flame bas been applied, and after trying all and every means of cleaning, I have finally. come back to aqua regia caused to boil at the dirty place and left in the tube for at least ten hours. Certainty of cleaning and jointing has led me to employ English flint glass exclusively in this work, and I always use the clean flame of an oxygen-gas blowpipe, which saves time so as to far more than repay the cost of the oxygen. All the blowing required in jointing the tubes is done through a compact but efficient establishment of filtering and phosphoric-acid tubes. The glass springs so extensively used throughout allow of considerable latitude in the relative movements of parts of the apparatus, and I have had no cases of spontaneous breaking. The whole of the glass work has been done by myself, as I can get no assistance of a suitable kind in Sydney.

Phil. Mag. S. 5. Vol. 35. No. 212. Jan. 1893. 
It may possibly be objected that some of the precautions taken are finnicking, and that (as in the manufacture of the oxygen) an unnecessary variety of absorptive materials are used. The reply I should make to such a criticism is that the complete operation of any one reagent is always to some small extent hypothetical, and I consider that by varying the reagents one helps to get rid of the chance of the persistence of impurities so small in amount as to be beyond the range of ordinary analysis, i.e. beyond the point up to which we are certain of the action of the reagents.

\section{Testing the Gas for Hydrocarbons and Hydrogen.}

In order to avoid the impurities introduced by the presence of internal electrodes, a tube was prepared with external electrodes. It was about 35 centim. long, of which length 20 centim. were made of capillary tube. In order to fill this with the pure gas, the apparatus was exhausted three times, as far as the chromous chloride U-tube, each exhaustion requiring seven or eight hours' work with the Geissler pump, after a good water-pump had got rid of the greater part of the gas.

The pressure of the remaining gas was imperceptible by inspection of the pump-gauge (of which a description will be given), but the pump continued on each occasion to show a just appreciable bubble of gas when the mercury flowed into the barometer-tube. The pumping was done at times separated from each other by three or four days in order to give the residual gas from the walls of the vessel time to come off ; of course this gas was removed as it appeared. The pressure I should judge was, on each occasion, about of the order of $\cdot 01$ millim. Several bottles of gas were also forced through the apparatus at the ordinary pressure.

Finally, on Feb. 19, 1891, the tube together with another for experimental purposes were fused off- the former at about -1 millim. pressure (the latter will be dealt with later). The tube had previously been heated till the glass began to soften and the walls to fall in, and the gas admitted had been for several days in contact with the phosphorus pentoxide. The only objection to the external electrode tube is that I have hitherto failed to produce a line-discharge in it-though of course Prof. J. J. Thomson's method (Phil. Mag. vol. xxxii. 1891) would give it in an endless tube without electrodes, and if the method had been known at the time I should have employed it. However, I got a bright bluish-white discharge in the narrow tube (the larger ends being golden), and this 
yielded a fine band-spectrum which has been described often enough, showing well both in the more and less refrangible ends. I took as my authority on the typical hydrocarbon bands those mentioned in the B.A. Report for 1880 , p. 226 .

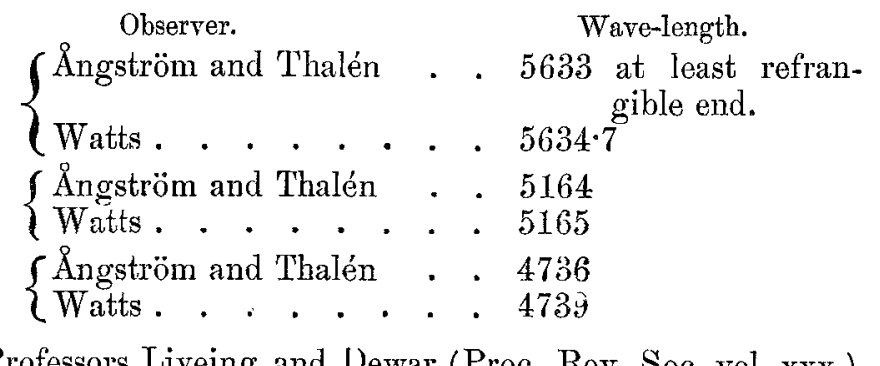

Professors Liveing and Dewar (Proc. Roy. Soc. vol. xxx.) say that the characteristic spectrum of hydrocarbons is composed (among others) of four bands in the orange, yellow, green, and olive. These were well seen by me in an olefiantgas tube prepared for the purpose without any pretensions to purity. The cone of a Bunsen flame was also examined, and I saw the lines (according to my map)

$$
\begin{aligned}
& \alpha \text { at } 5637 \text { instead of } 5634 \\
& \beta, 5164 \quad " \quad 5165 \text { (strongest) } \\
& \gamma, 4736 \quad, \quad 4738
\end{aligned}
$$

1 had a number of commercial tubes and examined these as a check; the following is the result:-

$\beta$ turned up in petroleum and alcohol but not in olefiant gas, where there was a band at 5197 to 5198 . This list band was strong and was seen in $\mathrm{CO}_{2}, \mathrm{H}, \mathrm{O}$, turpentine, petroleum, and coal-gas, as well as in the olefiant gas. I do not know what this band is due to, but it did not appear in the nitrogen. The nitrogen was examined side by side with an alcohol tube showing $\alpha$ and $\beta$ well ; but the former did not show these bands or any trace of them. The nearest band was at 5240, and quite too far away for there to be any mistake. The experiment was also tried with a similar tube and nitrogen at 10 millim. pressure, with a like result. Finally the examination was repeated at the lowest pressure at which I could get a brilliant discharge, which was of course far beyond the limit of discrimination of my pressure-gauge. The result was the same as before. The red and blue hydrogen lines were never seen in any of my tubes showing banded spectra. This analysis is of course dependent partly on the accuracy of our map, though when once the typical lines were picked up, their coincidence or not could be determined by direct expe- 
riment. It was necessary, therefore, to make a map of the spectrum to suit my spectroscope. The latter was an instrument with a 5-in. Trouton and Sims circle, and was made by Elliott Bros. ; one fine heavy glass prism was used throughout, and the map refers to it; it was always in the position of minimum deviation for each line observed. The mapping of the spectrum was done for me by Mr. J. H. D. Brearley (a student in the laboratory), who also assisted at this time in all the spectroscopic work and to whom my thanks are due. The map was made by a selection of 33 lines drawn from all sorts of sources, and verified in all sorts of ways, the tables used being those in the B.A. Reports for 1884, 1885,1886 ; Rowland's map (got in 1889) ; and Rowland's table, Pbil. Mag. 1889. Schellen was also found of some use. Each line was set upon ten times; and the prism was adjusted ten times.

Intermediate lines were now well picked up off Rowland's map. Our limit of discrimination was about 2 in the fourth significant figure of the wave-length, $i . e$. I could not decide with certainty that a line I made out to be at, say, 5165 was not at 5163 .

I append a list of the lines used in mapping the spectrum, not because it is in any way to be regarded as a model selection, but because I, as a beginner at spectroscopy, should have been very glad of such a list at the time, had I been able to find one. I also note the means adopted by us for the identification of the lines, as it may interest other beginners in spectroscopy. The lines are not given in the order in which they were examined, but in order of wave-length.

1.-B. Sun, 6867-796-6867-462. Rowland, Phil. Mag. June, 1889. Recognized by being near both to a large iron and to a potassium line.

2.- $\mathrm{Li}_{a}, 6705 \cdot 4$. From lithium chloride in a Bunsen flame. Wave-length from B.A. Report, 1884; given by Mascart, Liveing and Dowar, and Thalén.

3.-C. Sun (hydrogen). Good line recognized by comparison with a tube-spectrum of $\mathrm{H}_{2} \mathrm{O} . \quad \lambda=6563 \cdot 042$. Rowland, Phil. Mag. 1889.

4.-Sun, 6400.2. A good line picked up when the map was nearly finished from Rowland's map.

5.-Sun, 6191.7. Same as number 4.

6.- $\mathrm{Li}_{\tilde{\beta}}, 6102$. Authority, Mascart.

7.-Iron line, 5930.4. Rowland's map verified by comparison with spark-spectrum of iron. B.A. Report. Cornu gives $5929 \cdot 3$. 
8.-D. Halfway between the two lines. Mean, $\lambda=5893$. Rowland, Phil. Mag. 1889. Compared with sodium flame.

9.-Picked up from Chart. $\lambda=5709 \cdot 8$.

10.-Barium $(\gamma)$. From flame-spectrum of $\mathrm{Ba}\left(\mathrm{NO}_{3}\right)_{2}$. B.A. Report, 1884 . Liveing and Dewar. $\lambda=5534 \cdot 3$. A bright line.

11.-Magnesium, $\lambda=5528$. Rowland's map and sparkspectrum.

12.-Thallium ( $\alpha), \lambda=5349$. B.A. Report, 1885. Liveing and Dewar, and de Boisbaudran. Got from flamespectrum.

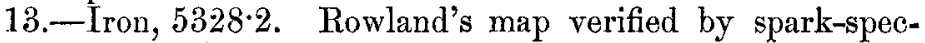
trum. A good line.

14.-E. $\lambda=5270$. Rowland's map, a big double. Picked up at first because it was found from Schellen's map that it was between two cobalt lines and near an iron line. These lines were seen in their proper relative places in the spark-discharge from iron and cobalt. This was one of the first lines identified.

15.- $b_{1}, 5183 \cdot 798$. Rowland, Phil. Mag. Verified by magnesium-spectrum. One of the first lines got.

16. $-\lambda=5041-5042$. Picked up from Rowland's map. Verified by finding from B.A. Report that it was an iron line and then confirming by spark-spectrum.

17. $-\lambda=4957 \cdot 6$. Picked up and verified as in the last case. 18. $-\lambda=4920 \cdot 6$. Ditto.

19.-F. $\lambda=4861 \cdot 492-4859 \cdot 929$. Rowland's map confirmed by vacuum-tube.

20.--Bismuth. $\lambda=4752$. B.A. Report, Thalén, and Huggins. From spark-spectrum. Not very good. Evidence as to identification, its general relation and appearance.

21.-Bismuth. $\lambda=4724 \cdot 5$. A very prominent line. Evidence and authority as in last case.

22.-Bismuth. $\lambda=4705$. Ditto. These lines cannot be mistaken.

23.-Strontium blue line. $\lambda=4607$. From flame-spectrum. B.A. Report, 1884. Liveing and Dewar, Thalén, Huggins.

24.-Cæsium $\beta$. $\lambda=4597$. B.A. Report, 1884; de Boisbaudran and Lockyer (4592). de Boisbaudran's value was taken, rather to my regret. One of the first lines got. Flame-spectrum.

25.-Cæsium $\alpha . \quad \lambda=4555$. Liveing and Dewar ; B.A. Report, 1884; Lockyer and de Boisbaudran (4560). Flame-spectrum. 
26.- Indium blue line. $\lambda=4509 \cdot 6$. Liveing and Dewar and Thalén, B.A. Report, 1884. Took Liveing and Dewar's number. Flame-spectrum.

27.-Solar spectrum. $\lambda=4405$. Rowland's map. Schellen's map gives it identical with an iron line. Verified by spark.

28.- Solar spectrum. $\lambda=4383.6$. As in case of last line (27).

29.-Solar spectrum. Hydrogen violet. $\lambda=4340 \cdot 5$. Rowland's map and vacuum-tube.

30.-Solar spectrum. $\lambda=4325 \cdot 982$. A fine line. Rowland's map and Phil. Mag. 1889. Picked up from map.

31.- Solar spectrum $G$. $\lambda=4308.023$. Rowland's map and Phil. Mag. The subject of a misprint in Rowland's list in Phil. Mag. 1889. It is an iron line.

32.-Solar spectrum. $\lambda=4227$. A calcium line given in Schellen's map. Well seen on Rowland's map. Could not verify from flame.

It will be found that the lines mentioned in this list are well spaced, and a curve drawn to them will enable most of the other lines to be set upon by calculation. It is obvious that all sorts of appliances must be to hend in order to verify the lines in the first instance, as well as a good stock of the rarer metals and their salts.

Preparation of Pump-Gauge.-All gauges of closed U-type. Gauge 1 constructed of tube 3 centim. in diameter, and 3 millim. thick in the walls, tested for evenness, \&c. Each limb about 20 centim. long, one limb closed at the top and the other open to the pump. The bend constructed of thick tubing having a bore of rather less than 1 millimetre in diameter. This gauge was boiled ont in a good Sprengel vacuum some twenty times, but I never could get rid of the last minute traces of gas. I have an idea that the last trace of gas cannot be got rid of - it can only be reduced asymptotically. However, the gauge was excellent; it was painted black, except for a sitit down each limb, and was read (with the precautions mentioned by Regnault) by one of the Cambridge Scientific Instrument Company's (Poynting's) cathetometers from about three metres' distance. This gauge behaved admirably, and $I$ used it during the spoctroscopic work; in some of the preliminary tube-experiments at a low pressure, however, a joint broke, and the momentum of the mercury was sufficient to break the closed end of the gauge in spite of the constricted bend. Under the circumstances, the gauge being much better than was required, I made No. 2 
similar to it, but of only 1 centim. diameter, and having a finer constriction of thermometer-tube in the bent part. This tube was not so good as the last, as it was not gas-free, and a small correction amounting to half a millimetre or so had usually to be applied on this account. This correction was determined several times, and a table of readings and corrections made out. There is no uncertainty as to the readings beyond about $\cdot 05$ millim., a quantity wholly insignificant for our purpose. This gauge lasted till 1892, when I finally got tired of applying corrections, and made a new and better gauge on the saine lines and of the same dimensions. This is still in use.

Tube Pressure-Gauge.-A glance at the Plate will show that this gauge was similar to the one used by Professor Thumson and myself in 1885, with the exception that it was of glass throughout. A tube of about 2 centim. diameter is bent into a U-shape, the bend being of tube about 5 millim. in diameter. The limbs are about 5 centim. long. The mercury is introduced so as to stand about halfway up the wide tuhes. 'The upper ends of the limbs are connected to each other, so that the gauge is really a "ring" space, and the space above the mercury communicates with the experimental tube by a filamentary glass tube of from one to two metres in length. When it is desired to observe the change of pressure in the spark-tube, the connexion between the space over the free surface of the mercury in the two limbs is interrupted by fusing a previously narrowed neck into a solid mass. This of course allows the pressure in the closed limb to retain its initial value, whatever that may be; while the pressure in the open limb varies with the pressure in the tube.

The effect of the metre-long filamentary tube is to hinder the diffusion of mercury vapour into the sparking-tube; and this in one or two experiments was also attended to by including a tube filled with clean silver foil between the mercury and filamentary tube. The filamentary tube, however, never let enough mercury-vapour in to give a mercury-spectrum at the lowest pressure at which I worked. Of course the mercury in the gauge was carefully boiled several times in order to cause any considerable quantity of gas that might have been condensed to be given off before the experiment began. These gauges, of which litrge numbers were made at one time and another, were very satisfactory, and when observed with proper precautions by means of the cathetometer, would certainly betray a difference of pressure of about $\cdot 05$ millim., all sources of uncertajuty being taken into account. 
Experimental Spark-Tuhe.-The first successful tube was furnished with external electrodes and sparked between Feb. 24 and March 2,1891. Details are (pressure 8.8 millim.) :-

h $\mathrm{m}$

Feb. 24. Sparked 130 Sparks being long and thin, large resistance in circuit.

Feb. 25. $\quad, \quad 5 \quad 0 \quad$ Ditto.

Feb. 28. " $\quad 3$ 0 Ditto. Then 3 hours 15 minutes with less resistance.

Mar. 2. " 40 The least perceptible discharge.

No effect was noticed at any time. The tube was left for three days in order that the temperature might reach the exact value it had when the gauge was senled, and it happened that this did not occur for three days. There was still no effect. This tube was sealed from the pump in series with the spectrum-tube and is still in my possession.

Tube 2.-After experimenting with many tubes mostly of the form shown in the diagram (fig. 1), with the armed ends in mercury cups, a satisfactory tube was gat on April 21st.

Fig. 1.

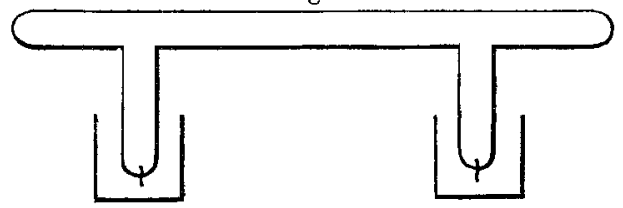

The difficulty arose from my determination to have the electrodes white hot for several hours by a preliminary sparking at about 1 millim. before the tube was finally filled with gas and sealed off. The excessive heating of the electrodes generally broke the glass. During this tine I noticed a thing which was new to me, but which I daresay is well known, viz., the ease with which the colour of the fluted spectrum-discharge may be made to change. Thus on the 20 th $I$ found that at a prossure of less than 1 millim., and with a very intense discharge from a coil giving 12-in. sparks without condenser, I could cause the golden-coloured discharge to become pinkish by heating the tube with a bare flame to about $200^{\circ} \mathrm{C}$., a necessary step in the preparation. The spectrum, of course, merely showed a shift in relative luminosity from the red towards the blue end. The action was reversed on cooling the tube. This could be repeated once or twice. However, with the next filling of gas the experiment did not succeed at all, no heating of the tube caused any change in the colour of 
the discharge; and in subsequent experiments only variable results were met with. The tube in question was finally sealed off at $9 \cdot 2$ millim., and sparked for six hours without effect. The next day the sparks were made stronger, and sent through the tube for four hours; still no effect was produced. The tube then cracked on the next attempt to spark it.

Tube 3.-April 25. Pressure $9 \cdot 4$ millim. In preparing the trube I noticed what I always noticed afterwards, viz., that at about 2-3 rillim. the discharge begins by being pink, and then in a minute or two becomes golden, and keeps so. On turning off the spark and reducing the pressure to about 1 millim., the same effect is observed. I have noticed this effect in all the tubes prepared since my attention was first directed to it. The sparking of this tube was as follows :-

April 25. 4 hours with the effective sparks described in the former paper. No effect.

April 27. 6 hours ditto. No effect.

April 28. 4 hours. Sparks of various intensity. No effect.

April 29. 4 hours. Ditto. Ditto.

This tube was sound throughout, and the result was convincing. It then occurred to me that possibly the mercury used in our gauges might account for the results obtained by Professor Thomson and myself in 1886. At that time we used a gauge fused directly on to the discharge-tube, with perhaps 10 centim. of 2-millim. tubing between the mercury surface and the tube, and with globules of condensed mercury hanging to the walls of the connecting-tube almost up to the discharge-tube itself. I remember that the glow often extended down to the mercury.

Tube 4.- Made with a side tube containing some very pure and dry mercury, so as to imitate our older form of tube. Pressure 8.5 millim.

June 11. Sparked 3 hours with effective sparks. No effect.

June 12. Sparked 4 hours with effective sparks. Examined on 15th, when it showed a just perceptible diminution of pressure.

June 15. Sparked 8 hours. Effect increased to about 1.2 millim. In order to try whether large sparks would reduce the effect, as was formerly found to be the case, I increased the intensity of the discharge, but did not succeed in producing any increase of pressure during half an hour (except that due to rise of temperature). The tube was then carefully examined, and it was found by holding a sheet of white paper 
behind it that there was a slight appearance of a bronzecoloured fog in the tube near the mercury-pocket.

June 16. A strong discharge sent through the tube so that it became warm to the hand, say $40^{\circ} \mathrm{C}$; the discharge was kept on for three hours.

June 18. The temperature again being the same as when the tube was sealed, it was noted that the effect appeared to have increased by about 22 millim. in spite of the strong discharge. Two "gallon" Leyden jars were then included in the eircuit provided with an air-gap, and parallel to the spark-tube. During two hours' sparking the effect went on increasing, and the bronze-coloured stain on the glass grew deeper near the drops of mercury till it became a black mirror.

This was of course partly due to the volatilization of the mercury near the drops. - The effect had increased to about 7 millim. by the next moraing, when the temperatures had become uniform.

June 19. Sparked, say, 5 hours. Effect on cooling was now $7 \cdot 3$ millim., indicating that only about 1.2 millim. of gas was left in the tube.

June 20. The mirror of mereury compound, which had now become very black about the narrow tube and in the part of the spark-tube near it, was heated with the Bunsen flame. After some heating, say at about $250^{\circ} \mathrm{C}$, the film began to crepitate audibly and emit flashes and sparks of blue flame. The deposit turned brownish as the temperature rose, the sides became indistinct, and finally the flash and crackling noise came on and the film vanished, leaving a clear bright tube. The process was repeated till the mirror of mercury had been chased all over the tube; and even then the gauge only came back far enough to show a pressure-difference of 4.2 millim. in the same direction as before- $-i$. e. it only came back by $3 \cdot 1$ millim. The ends of the tube, where the the electrodes were fused in, were not heated; and this may have accounted for the persistency of the diminution of pressure. In order to test this the tube was removed from the mercury cups and placed in an extemporized air-bath made out of a kerosene tin. Several thermometers were ranged alongside the tube, and the whole apparatus was covered by an asbestos eloth. The bath was heated by a Bunsen burner. A good many rather elaborate readings of tomperature and pressure were taken on June 20, the temperature rising to about $170^{\circ} \mathrm{C}$. in the middle of the tube and to a few degrees less at the ends. These readings turned out to have no significance, in consequence of a doubt 
as to whether the ends of the limbs of the tube into which the wires were fused were or were not at the same temperature as the rest of the tube. However, there is no doubt that the temperature of the ends was over $150^{\circ} \mathrm{C}$., and yet when all was cold again it was observed that the effect still amounted to a difference of pressure of 3.4 millim.

Now a compound of mercury and nitrogen, described as trimercuramine, was obtained by Plantamour (Ann. Chem. Pharm. xl. p. 115) by passing ammonia gas over mercuric oxide. This substance is said to explode on beating, and Plantamour gave it the formula $\mathrm{Hg}_{3} \mathrm{~N}_{2}$ on the strength of its behaviour with copper oxide in a combustion-tube, though he did not analyse it. The formula is, however, probably not very far from being correct. Plantamour does not state the temperature at which the nitride explodes, but says that if a little heap of the substance be placed on a card or piece of paper, and heated over the flame of a spirit-lamp, the explosion will occur before the temperature is high enungh for the paper to be turned yellow. My compound certainly required a higher temperature than this to start the explosion; but it is possible that its being in a nearly vacuous space, and very much contaminated with free mercury, may have had something to do with this. Plantamour's compound exploded with a white flame, which was bluish red at the edges; the compound I obtained emitted a brilliant bluish-white light on explosion. There is some evidence from Plantamour's paper that a temperature exceeding $200^{\circ} \mathrm{C}$. prevents the formation of the nitride from mercuric oxide and ammonia gas, and that the best temperature at which to carry out the synthesis is $150^{\circ} \mathrm{C}$. These facts appear to point to the conclusion that mercurv nitride is a dissociable compound; and the substance I obtained is probably so, as the continuation of the history of the tube will show. On June 22 the tube was wrapped in a good deal of copper gauze, and the bundle so made and into which three thermometers passed was placed in the air-bath. The burners were regulated so as to give a stationary temperature, first of $185^{\circ} \mathrm{C}$. and then of $196^{\circ} \mathrm{C}$. At the former temperature the gauge appeared to show that some substance was still undecomposed; but there is some uncertainty, from the ratio of the volume of the spark-tube to the gauge-tube not being exactly known. Estimating these volume-ratios, however, as nearly as possible by means of external measurement, and applying the necessary corrections, I found that the pressure ought to be (as shown by the gauge) 5 millim. greater on the tube side than on the sealed side. The observed pressure-difference was $5 \cdot 2$ millim.-a better agreement 
than one had any right to expect. The temperature was kept constant for a quarter of an hour after the observation, in the hope of detecting any further change, should it take place; but the readings remained constant. I a m therefore inclined to the belief that the substance dissociates on heating, and is all decomposed at a temperature of about $190^{\circ} \mathrm{C}$. On June $23 \mathrm{rd}$, when all parts of the apparatus were again at a uniforn temperature, the original effect had almost returned, there now being a difference of pressure of 3.2 millim. between the tube and the sealed space; or, in other words, partial recombination of the nitrogen appeared to have taken place.

Other matters required my attention at this time, and I did not get an opportunity to continue the study of the tube till August 13. On this day the pressure-difference was 3.7 millim., apparently showing that slow recombination had been going on. On August 13, 14, 15, and 17 the tube was heated each day up to about $190^{\circ}$, and curves drawn showing the relation between the pressure and temperature. These curves did not show a very good agreement, from the uncertainty as to corrections above mentioned ; and I therefore will only state that the effect of the heating on the 13th was to reduce the pressure difference to 2 millim., on the 14 th to 1.5 millim., on the 15 th to $\cdot 6$. On each day the heating took place during five hours or thereabouts, and the tube was maintained at its highest temperature for about an hour. On August 17 th the tube was exposed to a temperature of $200^{\circ} \mathrm{C}$. for six hours, and then kept at $110^{\circ}$ all night; and then, on August 18th, again heated to $200^{\circ} \mathrm{C}$. for five hours. When the tube was cold, on August 19th, the effect had entirely disappeared, the mercury standing at the same height in both limbs of the tube. On Angust 21st recombination appeared to have taken place to the extent of .5 millim., and then this disappeared again between August 21st and September 3rd. No further change having taken place by September 20th, the tube was finally disconnected, and the following conclusions were provisionally noted from a consideration of its behaviour.

1. A compound is formed when the electric discharge is passed over a mixture of mercury and nitrogen at a pressure of about 8 millim. The action commencing slowly, and increasing in velocity till it reaches a maximum, then decreasing, but continuing slowly till at least seven-eighths of the nitrogen is absorbed, the mercury being in excess.

2. The compound formed is brownish at first, but as time goes on it becomes black, probably owing to a continual increase in the quantity of mercury deposited with it. 
3 . The compound decomposes with crepitation at a temperature of about $200^{\circ} \mathrm{C}$. (very rough).

4. The compound is probably identical with the trimercuramine of Plantamour.

5. It behaves as a dissociable compound on heating and cooling, and can finally be entirely decomposed by repeating the heating and cooling often enough.

6. An attempt to get the dissociation curve with such accuracy as would allow the application of analysis failed, through the want of a preliminary determination of the relative capacity of tube and gauge.

It now became necessary to repeat the experiment under

Fig. 2.

To gauge, $120 \mathrm{~cm}$. of filamentary tube.

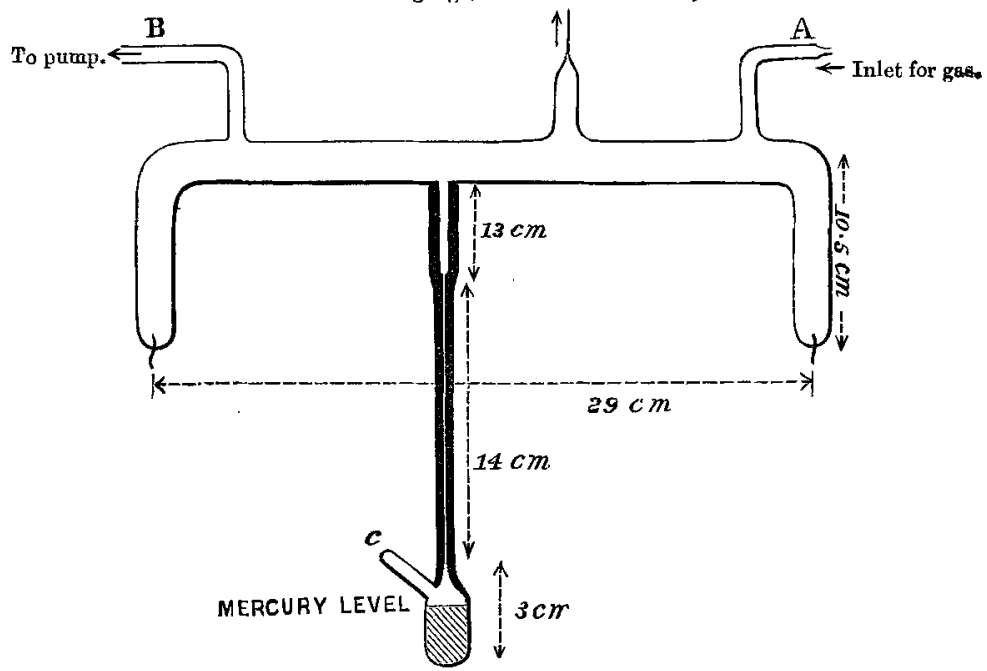

Inside diameter of main tube $1 \cdot 5 \mathrm{~cm}$. ; thickness of walls $\cdot 15 \mathrm{~cm}$.

slightly changed conditions, so that new facts might present themselves and the previous results be controlled. I decided to make a tube of such a kind that I could begin by sparking it so that the sparks only passed through nitrogen, and then, after a sufficient interval of time and at any convenient opportunity, introduce mercury with a view to observing the modified action. In order to vary the conditions, the whole drying apparatus up to the sulphuric-acid tube was pumped out afresh and filled with gas, which passed tbrough the reagents very slowly. This precess oceupied two days, one day and a half it took to exhaust the vesseis, and half a day 
(5 hours) for the gas to flow in. It will be convenient here to describe the tube employed, though the dimensions given are taken from a later experiment, which was supposed to be a repetition of the one in question; the dimensions of this tube may therefore be in error by a few per cent., but the actual dimensions are not of much importance. The ends of the tube dip down into mercury cups (not shown) so as to protect the part where the wires enter from leakage. The mercury pocket in the tube which forms the subject of the diagram (fig. 2) is attached to the spark-tube by means of a capillary tube 14 centim. long.

This capillary tube was the finest piece of thermometer tubing I actually happened to have by me, and was, as I should imagine, about $' 1$ millim. in diameter*. In preparing the tube after the final filling, the inlet at $A$ is fused off, and when the proper pressure is reached the pump connexion at B is sealed, and afterwards, in two or three hours' tjme, the connecting tube of the mercury gauge.

September 10-19. These days were spent in abortive attempts to get tubes of the pattern described to submit to baving their electrodes white-hot for two or three hours without fracturing the glass.

September 19. Tube 5, as described, sealed off at 8.7 millim. The mercury was put in hot through a side-tube, but was not boiled in situ in order to avoid getting vapour into the spark-tube. In order to avoid the presence of airbubbles between the mercury and the glass, the tube at $\mathrm{C}$ was at first about 8 centim. long, and was drawn down to a fine point, where it was fused off. The apparatus was then filled with nitrogen and well heated when containing a nitrogen vacuum, especial care being devoted to the mercury pocket. Nitrogen was then admitted at more than atmospheric pressure, and finally the end of the tube was broken off near $\mathrm{C}$ and the mercury run in as quickly as possible from a fine funnel reaching to the bottom of the pocket. The tube at $\mathrm{C}$ was then sealed, and the mercury warmed again to perhaps $100^{\circ} \mathrm{C}$. and the visible bubbles displaced, the tube being of course racuous.

Sept. 21. The mercury in the gauge was accurately at the same height in both limbs. Sparked for five hours with the best kind of sparks ; no effect.

Sept. 22. Sparked four hours with an air-break in circuit just sufficient to stop the spark but not the brush-discharge across the air-break. It was noticed that luminosity went

* This capillary tube was part of the later arrangements. In the tube under discussion its place was taken by a tube 2 millim. in diameter and only about 6 centim. long. 
down the tube to the mereury, and was much inereased by touching the outside of the tube near the mercury. This discharge, of course, was small compared with the discharge in the main tube. There was no effect on the gauge.

Sept. 23 . Sparked with rather large sparks $6 \frac{1}{2}$ hours. No effect.

Sept. 24. Sparked strongly, so as to make the tube warm, for $6 \frac{1}{2}$ hours. No effect.

Sept. 25. The mercury boiled up into tube. This led to the gauge showing a real increase of pressure of a little less than 1 millim.; I attribute this to gas from the sides of the tube between it and the mereury. Sparked strongly for $5 \frac{3}{4}$ hours; when all was cold the pressure excess had grown to about 2 millim., i.e. in a direction contrary to the one expected.

Sept. 26. Discharge on for $\frac{1}{2} \frac{1}{2}$ hours fairly strong, so as to warm tube perceptibly. The gauge then showed the silme pressure on each side.

Sept. 27. A wet paper band round that part of the tube where the mercury was most thickly condensed. Sparked four hours (?). No effect.

Sept. 28. Ditto 3 hours. No effect.

Sept. 29. Faint sparks for 5 hours ; wet paper band, as before. No effect.

Oct. 1. Temperature being more rigorsusly equal, and gauge carefully tapped, there is a shrinkage of about -1 millim. Sparked six hours with faint sparks and wet paper round tube. No effect

Oct. 2. Strong current on 6 hours. Looking the next day (Uctober 3rd), a faint yellow tinge was observed round the mercury drops, and the gauge showed $\$ 8$ millim. difference of pressure. Sparked hard 6 hours; the mercury was now obviously attacked, and when examined on October 3rd it was found that the pressure difference amounted to $3 \cdot 2$ millim. Sparked 3 bours with full discharge ; this increased the effect to 6 millim., and in another $4 \frac{1}{2}$ hours the discharge had become splendidly golden, the resistance in the tube small, and the tube was fluorescing strongly (a sign of mercury vapour). Effect increased to about 7 millim. The experiment was then interrupted by the cracking of the tube.

No opportunity for examining the deposit occurred till November 16 th, i.e. for more than a month. The iube was then cut into bits, and it was observed that the deposit had become partly incrusted with some whitish incrustation. On trying to produce an explosion the experiment failed, and it was obvious that the film had quite decomposed. It is to be noted that the mercury was only attacked with difficulty. 
Tube 6.-Dec. 5, 1891. This is the tube shown in fig. 2. All drying apparatus exhausted twice; tube prepared by a steady discharge during December 11th; left till 14th to test for leaks, when it was found that the whole apparatus was air-tight. Tube again sparked hard all December 14th with best pump-vacuum attainable. Electrodes at a bright red heat for $1 \frac{1}{2}$ hours ; mercury separated by long tube and boiled in pocket. Sealed off at about 6 millim. pressure on December 15 th.

Dec. 16. Sparked 2 hours through capillary water resistance ; tube slightly warm. No effect.

Dec. 17. Sparked so as to produce a yellow glow for 3 hours and by small blue sparks for 1 hour. No effect.

Dec. 18. Sparked with fairly strong discharge for 8 hours. Tube too warm to read : so left till December 31st, when it was found that the tube pressure had diminished by 22 millim. This led to an examination of the tube by hoiding a sheet of white paper bebind it; and it was found that a bronze-coloured film had formed where mercury had condensed near the top of the capillary tube. The mercury within the sphere of action was only the most minute trace. Sparked 3 hours and on.

Dec. 22. Effect about 3 millim. Sparked for about an hour, and then on careful examination it was found that the glow was actually passing down the tube to the mercury. It was clearly of no use to go on with the experiment in this way, and consequently the mercury was boiled into the tube and the spark turned on. The effect diminished during the boiling from $\cdot 35$ millim. to 3 millim. Sparked then for 5 hours ; effect increased to $1 \cdot 2$ millim.

Dec. 26. Sparked 4 hours. Effect $2 \cdot 4$ millim.

Dec. 27. Ditto 3 hours. Effect 4.3 millim.

Dec. 28. Sparked for one hour, when it was noticed that the tube-resistance had increased and the gauge showed 5.38 millim. of effect; hence the tube is nearly empty. On first turning on the spark, the illumination began by being strong for about one minute. The striæ were well marked, and the mercury spectrum prominent. After about one minate the luminosity diminished to that corresponding to faint blue sparks. In twenty minutes the tube was again hot and fairly luminous, and continued with luminous sparks and rising temperature for 35 minutes: the gauge showed that the rise of temperature (?) was producing an increase of pressure. Tube cooled for one hour, when it was found that the excessive sparking had at last cracked the tube near the platinum wire, and that some mercury had been drawn in. The tube was then cut into bits, and one was heated in a test-tube in a 
glycerine bath with the same precautions exactly as one uses in taking a melting-point. I extract the following account from my note-book verbatim:-_" I could not detect any change till about $230^{\circ} \mathrm{C}$., at which temperature quiet decomposition set in, and was complete and the tube clean at $235^{\circ}$. A considerable portion of the thermometer stem was outside the bath, though it did not begin to register till above $110^{\circ}$. Making all allowances, the temperatures might on this account have been anything less than $5^{\circ}$ above those given. The temperature rose very slowly, and the glycerine was kept well stirred by a crescent-shaped stirrer of zinc. Experiments on other bits of the tube were made by heating them in the bare flame. In two of these (out of about four) there was a distinct crepitation, but I did not note any flash, though that the decomposition when once started ran very rapidly over the whole nirror was well seen in all the experiments. The deposit suddenly brightened with a puff of vapour. I could detect no nitrous smell. The temperature must have been $200^{\circ} \mathrm{C}$. in all cases. The platinum deposits were enormous, and the electrodes torn up in all directions as described by Edison."

These experiments may be considered to settle the question of the cause of the effect observed by Professor Thomson and myself in 1885 and 1886. No trace of the effect was ever found unless the mercury was attacked; and, contrariwise, the mercury was never seen to be attacked without the "effect" making its appearance. It is curious to note the difference in the readiness with which the action can be brought about, even when all the cireumstances of the experiments appear to be identical. It is for this reason that I imagine that the reaction requires the intervention of mirute traces of some third substance, and the actual amount of these traces differs in different experiments. When the reaction once commences it goes on with increasing rapidity till near the end, and apparently faster the more powerful the discharge. .One experiment appears to indicate that even after the trimercuramine (?) is decomposed its constituents are in a state of "labile" equilibriam with respect to one another, and combine slightly or separate according to some set of circumstances which I cannot assign. It is curious, too, that the dissociation should be so irreversible. Unless the mercury vapour continually liberated is removed from the sphere of action-by combination with the platinum deposit, for instance-r cannot understand the effect of repeated heating; and, indeed, I consider that the suggestion just made is very possibly the true explanation.

The condensation of nitrogen, therefore, at ordinary temperatures has not been observed; but it is possible that it may

Phil. Mag. S. 5. Vol. 35. No. 212. Jan. 1893. 
occur at low temperatures. For this purpose I prepared

Tube 7, which is figured in the Plate in the position it occupied before it was sealed off. This tube was prepared with even more than the usual care. Thus, the cleaning of the tube occupied two days and the final rinsing was accomplished by means of some thrice-distilled water from the platinum and gold still. This water ran into the tube direct from the still. The electrodes were prepared by sparking during five hours with a discharge sufficient to make them redhot, during which time the tube was thrice filled and exhausted. The next day the tube was heated twice, and on each occasion about $500 \mathrm{cub}$. centim. of gas were passed through it; after which it was exhausted as far as possible (in an hour), and then the final charge was allowed to enter. The tube was sealed off at 8.7 millim. pressure, and then put in salt and pounded ice and kept at $-14^{\circ} \mathrm{C}$. by means of frequent stirring and the addition of fresh ice and salt. Sparked 2 hours with effective sparks : no effect. (The effect was looked for the next day, when the temperatures were equalized, but was not observed.)

Jan. 2. Sparked for 2 hours at about $-10^{\circ} \mathrm{C}$, all kinds of sparks, No effect.

The filamentary tube was then replaced by a wider one, as it was observed that the gauge only acted very slowly owing to the extreme fineness of the filamentary tube. The sparktube then required to be pumped out and prepared afresh, except for the heating of the electrodes, which, however, incidentally took place.

Jan. 4. Sparked $1 \frac{1}{4}$ hours at $-10^{\circ} \mathrm{C}$. No effect.

Jan. 6. Sparked 7 hours at a mean temperature of $-8^{\circ} \mathrm{C}$.; various sparks. No effect.

Jan. 7. Observed again, at very uniform temperatures. Still no effect.

Jan. 12. Ditto.

The conclusion is that no effect is produced on nitrogen, so far as condensation goes, by sparking it at a temperature of about $-10^{\circ} \mathrm{C}$. and at a pressure of $8 \cdot 2$ millim.

\section{Sunmary of Results.}

1. Nitrogen can be prepared by a continuous process in a state of great purity.

2. Nitrogen so prepared cannot be caused to condense by any available kind of spark down to a temperature of $-10^{\circ} \mathrm{C}$. and a pressure of about 8 millim.

3. When mercury is presen $i_{\text {, }}$ a compound of mercury and 
nitrogen is formed. This compound is probably identical with the nitride obtained by Plantamour.

4. The rate of formation of this nitride, other things being the same, is so irregular as to suggest that its formation is due to the interaction of an otherwise inappreciable trace of some other substance.

5. This compound is dissociable ; but under the circumstances of the experiment the dissociation process is not entirely reversible, since sufficient heating will permanently destroy the compound.

6. There are changes in the appearance of the discharge in nitrogen which can be brought about by very slight variations of temperature, and possibly of electromotive force, for which, so far as I know, no explanation is at present fortheoming.

Sydney, August 5, 1892.

II. On the Refraction of Rays of Great Wave-length in Rock-salt, Syluite, and Fluorite. By HeInRICH Rubens and BENJ. W. SNow*

\section{[Plate II.]}

N volume xl. of Wiedemann's Annalen one of the present 1 authors recently described a method whereby a knowledge of the dispersion of rays in the infra-red may be easily obtained. With the aid of this device the dependence of the index of refraction upon the wave-length was determined for sixteen materials; viz. for nine different samples of glass, for water, carbon disulphide, xylol, benzol, quartz, rock-salt, and fluorite. Inasmuch as in this paper is given a minute description of the methods employed, it will suffice here briefly to refer to the main features of the method of procedure followed in the present determination.

The rays from the zirconia burner of Linnemann, after being reflected from the front and the rear surfaces of a thin plate of air, enclosed between two parallel glass planes, were then concentrated upon the slit of a spectrometer, by which means two beams of light were produced, capable of mutual interference, so that the otherwise continuous spectrum of the incandescent zirconia plate was crossed by a series of vertical interference-bands. The wave-length $\lambda$ of each such dark band, multiplied by a certain whole number $m$, always equals the product of twice the thickness $d$ of the layer of air and the cosine of the angle of incidence $i$ of the rays. With the aid

* Communicated by the Authors.

$\mathrm{D} 2$ 